\title{
Disordered Mood in Cultural-Historical Context
}

\author{
Andrew G. Ryder ${ }^{1,2}$, Yue Zhao ${ }^{1}$, and Yulia E. Chentsova-Dutton ${ }^{3}$ \\ ${ }^{1}$ Concordia University \\ ${ }^{2}$ Jewish General Hospital \\ ${ }^{3}$ Georgetown University
}

\begin{abstract}
Mood disorders are observed worldwide and represent a major contribution to the global burden of disease. Despite the numerous ways in which these disorders vary across cultural contexts, however, the research literature is dominated by Western concepts. Cultural variations, and the ways in which they can best be understood and studied, comprise the focus of this chapter. We begin with a brief historical overview of disordered mood over different eras. Then, we turn to the contemporary literature on cultural variations in mood disorders, focusing on four domains: (1) etiological beliefs; (2) risk and resilience; (3) incidence and prevalence; and (4) symptom presentation. While many of the studies reviewed highlight intriguing differences across cultural groups, relatively few of them explore empirically the possible explanations for these differences. We thus propose an approach to understanding cultural variations in psychopathology based on a core idea from cultural psychology: the mutual constitution of culture, mind, and brain. Then, we briefly describe some of the ways in which symptoms of disordered mood can be understood as emerging from looping processes in the culture-mindbrain system. From this perspective, we conclude by looking ahead to some future directions for research. First, we emphasize the importance of integrative studies across culture-, mind-, and brain-levels. Second, we consider the possibility that historical changes in descriptions of disordered mood might go beyond shifts in our understanding to include culturally-shaped transformations in normal and abnormal experience.
\end{abstract}

Mood disorders disrupt lives worldwide, posing grave threats to productivity, relationships, and well-being. Depression in particular is projected to become one of the top two contributors to the global burden of disease by 2030 (Lopez \& Mathers, 2006). Mania, while less common, wreaks considerable havoc, often in tandem with depression. Given the personal and social costs of these disorders, there is an urgent need to better understand their etiology, presentation, course, and outcome, along with the best options for treatment. Yet, the overwhelming majority of studies on depression and mania, as with psychopathology more generally, rely on 'Western' models of conceptualizing, assessing, and treating these disorders. Even when some of these studies are conducted elsewhere, there is usually an assumption that Western-based criteria reflect universal phenomena. Cross-national research is thus most often conducted with the limited aim of evaluating people who meet these criteria in different cultural contexts. In this view, mood disorders are like other medical conditions that have a clear etiology and pathology leading to a specific set of symptoms. Available evidence suggests otherwise.

In this chapter, we argue that mood disorders are profoundly shaped by their cultural and historical context. We begin with a brief history of mood disorders before turning to contemporary research on: (1) etiological beliefs; (2) stress and vulnerability; (3) incidence and prevalence; and
(4) symptom presentation. As this literature includes many more studies of depression than mania, and many more studies conducted in North American or Chinese samples compared with the rest of the world, our review is weighted accordingly. We note also that while these bodies of research inform us about how mood disorders vary across cultural contexts, relatively little is understood about how culture shapes these disorders.

Indeed, throughout our review we are hampered by the lack of a coherent view of culture in many of these studies. Moreover, there is a paucity of theoretical and empirical work integrating the cultural level of analysis with those of mind and brain. We are left with an isolated set of cultural facts about the mood disorders rather than an integrated perspective. In the second half of this chapter, we therefore begin outlining what such a perspective might look like in light of emerging ideas on the mutual constitution of culture, mind, and brain (Kitayama \& Uskul, 2011; Ryder, Ban, \& Chentsova-Dutton, 2011). We conclude by briefly considering two directions for future research. For the first, we call for more studies explicitly designed to study the interactions of culture-, mind-, and brain-levels of analysis. For the second, we return to the historical review of the mood disorders and propose that historical era, like cultural context, might shape not only our understanding of these disorders but also the disorders themselves. 


\section{A Brief History}

Descriptions of various dejected and excited states can be found in Western literature dating back to its written origins (Jackson, 1985). The concept of melancholia, for example, can be traced to the writings of Hippocrates, who described symptoms that include despondency, loss of appetite, insomnia, irritability, and restlessness. Indeed, the term itself comes from Hippocrates' humoral theory of illness, with melancholia describing an excess of black bile (Ancient Greek melan- = black, kholē = bile); excited mania, meanwhile, was attributed to an excess of yellow bile. Aretaeus of Capodoccia, in the second century C.E., described how some people tend to oscillate between excited and melancholic states (Akiskal, 1996).

Whereas melancholia described a psychological state with a biological cause, acedia described a superficially similar state with a very different etiology. The term was originally used to describe the sense of isolation and spiritual despair experienced by some ascetic monks, and included experiences such as sad mood, low spirits, anguish, loss of energy, fatigue, carelessness, sloth, and negligence. These 'symptoms' were seen as the consequences of succumbing to the demon of acedia - the "noonday demon" - sent to test the devoted monk (Wenzel, 1960). Over time, as various schemes of deadly sins were described and preached against, acedia became associated with these universal dangers (Jackson, 1985). While still understanding acedia as a sin, the scholastics of the 1200s increasingly described it as a disorder of emotional life, sometimes even in humoral terms. Notably, texts that considered natural causes also tended to describe acedia less as a vice, reducing its stigma (Wenzel, 1960).

By the 1500s, acedia had been absorbed by the humoral theory, losing its distinct etiology but retaining some of its moral force (Kleinman, 1986). Meanwhile, melancholia became an increasingly broad term so that by the early 1800 s it included most mental illnesses that involved few delusions as opposed to many. This trend towards generality started to reverse with "Faculty Psychology", a 19th century philosophical attempt to break down psychological functioning into irreducible 'faculties'. The faculty of 'affectivity' captured the domain of mood and emotion; disorders in this faculty were referred to as affective disorders. The conceptualization of melancholy thus shifted from a very broad category to a specific form of disordered mood. Borrowing a then-popular word in cardiovascular medicine for reduced function, this new disorder was named depression (Berrios, 1996).

The classical idea that depressed and excited mood states might be linked had lain dormant for almost two millennia, but was picked up again in the mid-nineteenth century. In the 1820 s and 1830 s Esquirol, with his students and colleagues, began to follow the course of mental illness over time rather than relying on cross-sectional descriptions (Akiskal, 1996). By the time of Kraepelin's (1921) landmark division of schizophrenia and manic-depressive illness, both 'mania' and 'depression' clearly defined serious, potentially debilitating, problems. What then of the milder forms of depression, more in the tradition of acedia?

While European psychiatrists were linking mania and depression, American neurologists were describing various syndromes attributed to functional exhaustion of the nervous system, or neurasthenia (Shorter, 1992). Beard (1869) described a nervous syndrome of more than 50 symptoms, including "general malaise, debility of all the functions, poor appetite, abiding weakness in the back and spine, fugitive neurological pains, hysteria, insomnia, hypochondriasis, disinclination for consecutive mental labor, severe and weakening attacks of sick headache, and other analogous symptoms..." (p. 12). Nervous exhaustion was most commonly described among educated people in the middle and upper social classes, particularly 'mental workers' who were dealing with the stressful consequences of rapid modernization (Liu, 1989). By the dawn of the twentieth century, neurasthenia was well known both professionally and popularly throughout the industrialized West.

The twentieth century also brought psychoanalysis. While the early analysts classified neurasthenia as an 'actual neurosis' - that is, caused by a physical problem of the nervous system - the spread of psychoanalytic practice was accompanied by the spread of its conceptual domain. As it became increasingly difficult to diagnose neurasthenic symptoms in the absence of a putative intrapsychic conflict, the category fell into disuse (Chatel \& Peele, 1970). Ironically, now that psychiatry has all but abandoned this archaic term, other branches of medicine are confronting relatively 'new' problems characterized by prolonged fatigue. Abbey and Garfinkel (1991), for example, found a high degree of overlap between Chronic Fatigue Syndrome and the original descriptions of neurasthenia by Beard.

Curiously, as the neurasthenia diagnosis was all but disappearing in North America and Western Europe, it was being introduced, studied, and increasingly adopted in East Asia. Between the founding of the Republic of China in 1912 to the Communist Revolution of 1949, Chinese physicians used the American concept of neurasthenia, regarding it as an anxiety reaction (Liu, 1989). After the revolution, the chief scientific influence on China shifted to the Soviet Union but there too, neurasthenia had become popular in no small part due to the integration of Beard's (1869) original work into Pavlovian psychology. After China's break with the Soviet Union in the 1960s, Chinese physicians took a renewed interest in the indigenous literature on Traditional Chinese Medicine (TCM), noting several similar, popularly known, and non-stigmatizing diagnostic equivalents to neurasthenia in the TCM system (Lee, 1999). While these equivalents have an ancient pedigree within TCM, constructs resembling depression emerge much more recently and never become as 
important as in the West (Tseng, 1974).

In many ways, Chinese neurasthenia appears to be following the trajectory of its Western counterpart. In the 1980s, neurasthenia rates were extremely high, although the label may have been assigned in many cases to help patients and families avoid stigma (Lee, 1999). Over the past few decades, however, there has been a steady shift towards the use of the 'depression' label, and perhaps even a shift towards a symptom presentation pattern that more closely resembles Western depression. This intriguing possibility, that the historical era can actually shape symptom presentation and not merely how syndromes are described, will be taken up in the final section of this chapter. First, let us consider some contemporary evidence pertaining to cultural group variations in depression and mania.

\section{Cultural Group Variations}

Research on mood disorders is based almost exclusively on one of two widely used classification systems - the Diagnostic and Statistical Manual of Mental Disorders (DSM-5; American Psychiatric Association, 2013) or the International Classification of Diseases (ICD-10; World Health Organization, 2008). Both systems define pathological disturbances of mood as central to mood disorders. As described in chapter 2 of this volume, this family of diagnoses can be broadly subdivided into the unipolar mood disorders, such as Major Depressive Disorder (MDD) and the bipolar mood disorders, such as Bipolar I Disorder (BD). Indeed, 'depression' can be used to describe a mood state, a symptom, a constellation of symptoms, a discrete episode, a chronic condition, a trait-like dimension, or a categorical diagnosis.

In addition to this ambiguity, the term 'depression' carries with it a set of cultural assumptions. For that reason alone, a straightforward comparison across different cultural groups based on a formal diagnostic system is problematic. Even a diagnostic category that appears to work well enough in a given cultural context does not necessarily carry the same meaning, or describe the same kind of people, as it does in another cultural context. Diagnostic manuals are themselves cultural products. The centrality of depressed mood and anhedonia to MDD in a given context, for example, cannot emerge as a finding from research using a diagnostic system that already requires one or both of these symptoms. Indeed, while in this chapter and elsewhere we retain terms like 'depression' and 'bipolar disorder' when reporting results from studies grounded in these systems, we nevertheless prefer when possible to use broader and less explicitly culture-bound terms such as 'serious distress' or 'disordered mood' (Ryder \& Chentsova-Dutton, 2015). The available research evidence bolsters our concern about the uncritical use of Western labels - and Western ideas more generally-to describe mood disorders across cultural contexts. It is to this evidence that we now turn.

\section{Etiological Beliefs}

The criteria for mood disorders in the DSM and ICD reflect Western beliefs and values that emphasize the Cartesian distinction between mind and body (Ryder et al., 2008; Ryder \& Chentsova-Dutton, 2012). Indeed, most of the scientific and clinical literature on mood disorders has been generated in contexts with strong cultural links to Western Europe, particularly the United States. Many of the basic assumptions of these contexts are, however, idiosyncratic from a global perspective. Researchers have exhaustively documented the emphasis in the industrialized West on the uniqueness and autonomy of the self; in this view, healthy functioning is grounded in a positive self-image, personal values and achievements, and open expression of one's emotions (Kirmayer, 2007). In such contexts, mental disorders are often 'psychologized', understood as problems of the mind (Ban, Kashima, \& Haslam, 2012).

Far more common globally are cultural contexts that promote interdependence through relationships and social networks, although there is considerable heterogeneity in the ways this is done. In East Asian cultural settings, for example, healthy functioning tends to be characterized by selfcriticism, avoidance of interpersonal conflicts, and inhibition of emotional expression depending on relational needs, all with the aim of preserving group harmony (Heine, Lehman, Markus, \& Kitayama, 1999; Markus \& Kitayama, 1991, 2010). Mental disorders are more likely to be flagged if they disrupt this harmony and then 'moralized', understood as a moral violation or failure of some kind (Ban et al., 2012). The consequence may be increased stigma for certain kinds of symptoms, and a tendency to avoid discussion of these symptoms: for example, stigma was associated with psychological symptoms but not somatic symptoms in a sample of female Turkish migrants in Germany (Montesinos et al., 2012).

In many cases, more than one model of serious distress may be available in a given cultural context, with use of these models depending on the situation. Pritzker (2007), for example, observed that depressed Chinese people often shift in their use of bodily metaphors, locating depression sometimes in the heart and sometimes in the brain, each with different implications. Modernization and Westernization processes in many societies can introduce people to new models of depression, but these models often end up co-existing with - rather than replacing - traditional models. Privileging some expressions and ignoring others can lead researchers and clinicians to miss important heterogeneity within a particular cultural context.

This diversity of beliefs, both within and across cultural contexts, also has consequences for the placement of the dividing line between normal and abnormal; in other words, at what point is serious distress pathologized? In some cultural contexts, serious distress is understood as a troubling but 
otherwise normal part of the human experience. In Good's (1977) classic ethnography conducted in Iran, serious distress was seen as the consequence of living a moral life in a fallen world. People in Eastern European cultural contexts tend to report lower levels of positive emotions compared to other Europeans, but they do not pathologize this tendency unless the symptoms are severe (Jurcik, Chentsova-Dutton, Solopieva-Jurcikova, \& Ryder, 2013). Similarly, compared to their peers in the United States, older Russians are less likely to view depression as an illness and more likely to consider it a normal part of aging (Turvey et al., 2012). Similar conclusions have been drawn by researchers studying older Hmong and Cambodian samples (Lee et al., 2010). Cultural variations in etiological beliefs about serious distress can shape understandings about who is potentially vulnerable, as well as the kinds of stressors that might be especially troublesome. These beliefs, as we shall see, might even act directly to shape risk for serious distress in particular cultural contexts.

\section{Stress and Vulnerability}

There has been a long-standing interest in the interactional contributions of stress and vulnerability on risk for mood disorders. Certain vulnerability factors are potentially universal. For example, rumination, hopelessness, and reliance on maladaptive coping strategies are associated with increased depression in both Chinese and North American samples (Auerbach, Abela, Zhu, \& Yao, 2010; Stewart et al., 2004). Nonetheless, there is evidence to support the cultural shaping of vulnerability and its sources. For example, emphasizing individualistic values may confer vulnerability to depression by broadening discrepancies between realistic outcomes and cultural ideals, in turn leading to negative self-evaluation (Reynolds, Stewart, MacDonald, \& Sischo, 2006). Submissive behaviors can exacerbate this vulnerability (O'Connor, Berry, Weiss, \& Gilbert, 2002), perhaps attributable to the tendency in Western cultural contexts to emphasize self-assertion (Heine et al., 1999).

There is emerging evidence that the high value placed on the pursuit of happiness, so central to American individualism, may actually confer vulnerability to mood disorders. For example, evidence from non-clinical samples show associations between happiness valuation and loneliness, unhappiness, and less response to a positive mood induction (Mauss, Tamir, Anderson, \& Savino, 2011; Mauss et al., 2012). Happiness valuation was also associated with more depressive symptoms in a remitted depressed group and was stronger in a remitted depressed group compared to a nondepressed group (Ford, Shallcroft, Mauss, Floerke, \& Gruber, 2014). Moreover, happiness valuation was associated with a measure of BD risk, higher likelihood of past BD diagnosis, and worse prospective illness course for BD (Ford, Mauss, \& Gruber, 2015). These findings may be context- specific: in a four-country study, happiness valuation was only associated with lower well-being in the United States (Ford et al., 2015).

Culture also shapes both the likelihood and assigned meaning of stressors. For example, interpersonal conflicts appear to be primary stressors for Western adolescents (Grant et al., 2006), whereas poor academic performance has a stronger association with depression in Chinese versus American adolescents (Li \& Zhang, 2008). Poor academic performance was found to be prospectively associated with depression in Chinese children, and to predict severe depression and suicidal ideation in Chinese adolescent samples (Chen, Rubin, \& Lee, 1995; Hesketh, Ding, \& Jenkins, 2002). Auerbach, Eberhart, and Abela (2010) found that interpersonal stress interacted with low self-control to predict a later increase in depressive symptoms in Canadian but not Chinese adolescents. The authors propose that stressed Chinese adolescents with reduced sense of control tend to act in ways that generate future stress. As culture shapes importance of primary control (Sastry \& Ross, 1998), future studies should evaluate cultural mechanisms underlying these findings. Such mechanisms might help explain crosscountry group differences reported in epidemiological studies.

\section{Incidence and Prevalence}

Further evidence suggesting the importance of cultural context to mood disorders can be found in cross-national projects by psychiatric epidemiologists. While these studies are limited by their tendency to assess mood disorders according to Western-derived criteria, country-level differences at least alert us to the possibility of intriguing cultural variations. Weissman and colleagues (1996) examined ten sites and found lifetime prevalence estimates ranging from $1.5 \%$ (Taiwan) to $19.0 \%$ (Lebanon). Studies by the International Consortium of Psychiatric Epidemiology also covered ten sites and found lifetime prevalence estimates ranging from $3.0 \%$ (Japan) to $16.0 \%$ (United States) (Andrade et al., 2003).

Researchers have generally found lower rates of depression in East Asia, relative to North America and Western Europe. For example, the Global Burden of Disease project reported a 1- year incidence rate for MDD in China of $2.3 \%$ (Murray \& Lopez, 1996) compared with the 10.3studies, the 1-year incidence rate for MDD ranges from a low of about one in forty people in some countries (e.g., in East Asia) to a high of about one in twelve in others (e.g., in North America) (e.g., Demyttenaere et al., 2007; Kessler et al., 2009; Lin \& von Korff, 2008; Ohayon \& Kong, 2006; Vasiliadis, Lesage, Adair, Wang, \& Kessler, 2007).

Weissman and colleagues (1996) found that the lifetime prevalence of BD ranges from $0.3 \%$ (Taiwan) to $1.5 \%$ (New Zealand). In other words, a Western society had a rate 
five times higher compared to a Chinese society (the United States was three times higher than Taiwan), raising questions about the cultural shaping of BD similar to those about MDD (Hechtman, Raila, Chiao, \& Gruber, 2013). A recent Chinese epidemiological study found the 12-month prevalence rate of BD to be $0.1 \%$ (Shen et al., 2006); in contrast, the U.S. National Comorbidity Survey Replication found a one-year prevalence rate for $\mathrm{BD}$ of $2.6 \%$. The reasons underlying this variation are unclear, and not necessarily related to 'culture' as such. For example, Noaghiul and Hibbeln (2003) have shown an inverse relation between bipolar rates and seafood consumption, suggesting a link with Omega 3 fatty acids. Other researchers have found evidence for higher rates of hypomanic traits, which are associated with increased risk for mood disorders, in at least some migrant samples (Carta et al., 2012; Swinnen \& Selten, 2007). Evidence of group difference is not necessarily evidence for cultural shaping, underscoring the need for studies specifically designed to unpack these effects.

\section{Symptom Presentation}

In contrast to syndrome-focused epidemiological studies, research on symptom presentation has focused much more directly on cultural processes. There is now considerable anthropological and psychological evidence to support the idea that culture shapes not only beliefs about serious distress, but also the symptoms themselves. While the list of potential symptoms across cultural contexts is finite, it is also much longer than the collections presented in DSM or ICD; culture appears to play a critical role in determining which symptoms are emphasized (Ryder \& Chentsova-Dutton, 2015). For example, Korean migrant women in the U.S. tend to emphasize emotional entrapment - the experience of having to hide one's negative feelings about others without an outlet for expressing them (Bernstein, Lee, Park, \& Jyoung, 2008). Distressed Puerto Ricans tend to focus on crying jags, sleeping difficulties, and even visions (Koss-Chioino, 1999), whereas distressed rural Nepalese tend to complain of numbness and tingling (Kohrt et al., 2005).

One of the few cultural variations in the mood disorders that has received sustained research attention from multiple research teams over several decades centers on 'Chinese Somatization' - the tendency, relative to Western norms, to de-emphasize psychological symptoms of depression in favor of somatic symptoms (Ryder \& Chentsova-Dutton, 2012). The first studies on this topic were published in the early 1980s, and contrasted low rates of depression with high rates of neurasthenia. In a now-classic study combining anthropological and psychiatric methods, Kleinman (1982) studied 100 consecutive neurasthenia patients and found that most of them could be diagnosed with a depressive disorder based on a structured interview. Nonetheless, he noted that many of these patients differed from a typical American pre- sentation; chief presenting complaints included headaches (90\% of cases), insomnia (78\%), and various pains (49\%), whereas depressed mood was not often a primary concern $(9 \%)$.

Although many papers in subsequent years would attempt to explain the Chinese tendency to emphasize somatic symptoms, to our knowledge the first direct comparisons of Chinese and Western samples did not take place until almost two decades later. Several studies were conducted during this time to support Chinese somatization. They tended, however, to interpret Chinese findings in light of the standard Western clinical picture of depression as predominantly psychological (Ryder \& Chentsova-Dutton, 2012). This bias became increasingly problematic in light of growing evidence that somatic symptoms are ubiquitous in depression, including in Western cultural contexts (Isaac, Janca, \& Orley, 1996).

An international multisite study confirmed that somatic symptoms are indeed found worldwide; at Western sites, predominantly somatic presentations accounted for between 45\% (Paris, France) and 95\% (Ankara, Turkey) of patients meeting criteria for MDD, with a rate of $87 \%$ in Shanghai, China (Simon, von Korff, Piccinelli, Fullerton, \& Ormel, 1999). The authors of this study argued that cross-site variation was best explained by the extent to which patients at each site had the opportunity to establish a personal relationship with their clinician, rather than by the cultural context per se. Yen, Robins, and Lin (2000) reported that Chinese patients seeking treatment at counseling centers and clinics emphasized somatic symptoms relative to Chinese students, but found no differences when comparing Chinese students with American students. These authors concluded that a somatic symptom emphasis might be attributable to the 'sick role' played by Chinese patients.

The story was different when clinical samples were directly compared cross-nationally on a symptom-by-symptom basis. Somatic chief complaints were much more common in depressed Malaysian Chinese outpatients compared with depressed Euro-Australian outpatients. A self-report measure also showed a greater emphasis on somatic symptoms in the Malaysian Chinese sample, along with an even more striking emphasis on psychological symptoms in the Euro- Australian sample (Parker, Cheah, \& Roy, 2001). A follow-up study with Chinese-Australians in a primary care setting found that symptom presentation increasingly matched Euro-Australian norms over time, consistent with the view that these patterns are attributable to the specific cultural context (Parker, Chan, Tully, \& Eisenbruch, 2005). Ryder and colleagues (2008) replicated these findings in Chinese and Euro-Canadian depressed outpatients using clinical and 16 structured interviews along with questionnaires. Again, group differences were particularly striking for psychological symptoms.

Findings from a small set of studies suggest that there may be cultural variations in the clinical presentation of $\mathrm{BD}$ as 
well. Some of these studies evaluate the degree to which BD emphasizes mania versus depression. Unipolar mania with no depressive episodes has been observed both among the Chinese (Lee, 2001) and the Yoruba in Nigeria (Makanjuola, 1985). Similarly, BD patients of African origin were more likely than White Britons to show exclusively manic presentations (Kirov \& Murray, 1999). In a study of BD patients in Israel, a predominance of manic episodes over depressive episodes was observed, in contrast to the pattern commonly observed in European patients. As with epidemiological findings, differences identified in particular countries are not necessarily due to culture as such: the authors propose that their Israeli findings may be due to the influence of temperature and exposure to sunshine (Osher, Yaroslavsky, el-Rom, \& Belmaker, 2000).

There is also evidence of variability in mania symptoms. Among the Amish, high levels of persecutory delusions and grandiose themes, particularly with religious overtones, were observed in an interview-based study (Egeland, Hostetter, \& Eshleman, 1983). A low occurrence of flight of ideas and a relatively high occurrence of persecutory and self-blaming delusions were found among manic patients in eastern India (Sethi \& Khanna, 1993). Afro-Caribbeans were more likely to have had mood-incongruent delusions and lower rates of suicidality compared to White Britons (Kirov \& Murray, 1999). Unfortunately, most of these studies fail to test potential explanations for why these group differences are observed.

In summary, there are clear indications of variability in clinical presentations in different cultural contexts. However, there is little empirical evidence pertaining to the reasons for these 17 variations. Our consideration of the research literature on the cultural shaping of mood disorders has now brought us to the point where a deeper integration is needed. It is all too easy to present a set of disconnected findings from a range of cultural contexts without saying much about culture, and how it actually shapes mood disorders. We thus turn now to a brief consideration of culture, and its deep interconnectedness with mind and brain, before considering some potential research directions that could emerge from this perspective.

\section{Where is 'Culture' in Cultural Studies of Mood Disorders?}

\section{The Mutual Constitution of Culture, Mind, and Brain}

Although we are influenced by several disciplines concerned with the link between culture and mind, we emphasize a cultural psychology approach. Drawing from anthropology, cultural psychology has increasingly adopted a distinction between 'culture' and 'cultural group'. We rely on provisional cultural groups because they facilitate research design and communication, not because they have some kind of fixed essence. Far from fixed, culture is fluid, partial, hybrid, and contested. Work on distributed cognition demonstrates that cultural meanings do not have to be accessible to everyone equally, or at all (Sperber \& Hirschfeld, 2004). Individual group members may fully or partially adhere to, flexibly adapt, or fully reject a given culturally normative practice. Culture can neither be reduced to mind, nor mind to culture - rather, they exist in a dynamic relation of mutual constitution (Shweder, 1990).

Similarly, we must resist a growing tendency in psychology and psychiatry to view mind and culture as reducible to the brain, as there are limits to the explanatory power of theories that invoke only brain processes. Recent developments in cognitive science characterize the mind as embedded in the local social world, and even the physical environment (Hutchins, 2011). In keeping with contemporary cultural psychology, we therefore propose that the brain is an integral part of any holistic systems view of human psychology (Kitayama \& Uskul, 2011). The brain is vital to our perspective not because it is a fixed entity from which all causal arrows proceed, but precisely because it is evolutionarily adapted and environmentally responsive, flexible and also constrained, all at once. Mutual constitution must be extended to include the brain, but not at the expense of the mind (Ryder \& Chentsova-Dutton, 2015).

Treating culture-mind-brain as a single, multilevel system has implications that go beyond the traditional tripartite division provided by the biopsychosocial model. To begin with, we cannot easily compartmentalize even specific claims about a given disorder to a single level, and we must avoid the easy reduction of mood disturbance to the 'lowest' level. Instead, events cascade through culture-mind-brain in all directions. Rapid socioeconomic change can lead to job loss and geographical dislocation, which in turn can stress family systems, making people feel miserable and impacting their cortisol levels - which itself has further consequences, for brain functioning, subjective experience, family dynamics, and so on.

In previous work, we have proposed that one way of simplifying matters, while still attending to all levels of culturemind-brain, is to adopt the concept of cultural scripts (Ryder \& Chentsova-Dutton, 2015). Normative cultural scripts can be understood as a set of interpretative lenses that provide the backdrop against which symptoms are experienced. For example, depressed European Americans are less emotionally reactive - and depressed Asian Americans are more emotionally reactive - than their non-depressed counterparts (Chentsova-Dutton et al., 2007). In one sense, these are opposing findings; in another, both findings show that depression is associated with emotional reactivity that differs from relevant cultural norms. But this finding raises an important question: different with respect to which norms? 


\section{The Emergence of Emotional Symptoms in Culture- Mind-Brain}

Certain experiences deviate sufficiently from local cultural norms that they are seen as abnormal, but nonetheless there are consensual understandings of how these experiences should be construed. Moreover, not only do these deviant cultural scripts help the sufferer make at least partial sense of his or her suffering, there is emerging evidence that they guide people to attend to particular experiences, and may even contribute to their emergence as problematic symptoms rather than transitory events. For example, a person concerned about a relapse into depression may scan their thoughts for self-doubt, making these thoughts more likely to occur. Meanwhile, all kinds of other thoughts, emotions, sensations, and other potentially symptomatizable experiences fade into the background inasmuch as they do not fit available interpretive lenses (Kirmayer \& Sartorious, 2007; Ryder \& Chentsova-Dutton, 2015).

The attention-directing effects of these scripts do not cease with the emergence of a symptom or set of symptoms. Rather, identification of a symptom can elicit further interpretations, which can trigger emotional responses, which themselves are accompanied by a set of potential somatic, cognitive, and behavioral consequences. Pathology emerges as the consequence of looping effects where the response to a particular experience further exacerbates it (Kirmayer \& Sartorious, 2007). These short-term loops are joined by longerterm loops, which can serve to maintain and exacerbate the pathology. Indeed, looping may be central to why transient symptoms develop into more chronic syndromes (Ryder \& Chentsova-Dutton, 2015). Symptoms that frequently get pulled into these kinds of chronic loops within a given cultural context are likely to be named, and identified as both important and problematic within that context - and in Western contexts, perhaps granted status as a psychiatric category.

\section{Rethinking Research on Mood Disorders}

If we take the view that mood disturbances emerge and are maintained at least in part by looping effects spanning culture, mind, and brain, there are some implications that might change the way we do research. At a minimum, we hope that these changes will include a thoroughgoing engagement with the mechanisms underpinning cultural variation, with a move away from simply cataloguing and speculating on group differences. We thus conclude with a brief look at two promising research directions that could help push the field forward-integrative research on culture-mind-brain and the psychological study of historical change.

The premise that culture-mind-brain constitutes a single, multi-level, system can help inform recent work on the biological and cultural co-construction of mood disorders. For example, vulnerability to depression is associated with sen- sitivity to environmental stressors, which in Western cultural contexts is associated with variation in one of the serotonin transporter genes (Caspi, Hariri, Holmes, Uher, \& Moffitt, 2010). From a global perspective, however, populations with higher rates of this genetic sensitivity actually show lower rates of depression. Chiao and Blizinsky (2009) present evidence that people from ethnic groups with a higher likelihood of environmental sensitivity tend to inhabit cultural contexts that foster collectivistic values, which may protect people in these groups from serious distress.

In short, concern for the cultural should not be opposed to the many exciting developments in clinical neuroscience. Researchers should at least be aware of relevant research targeting all three levels; moreover, we suspect that truly transformative work will be highly integrative. For example, Kim and colleagues (2010) have demonstrated that engagement in emotional support-seeking is best predicted by interactive effects between Korean vs. Euro- American group membership (culture), subjective distress (mind), and oxytocin receptor polymorphism (impact on brain). Immordino-Yang, Yang, and Damasio (2014) present evidence that cultural group (Chinese vs. Euro-American) is associated with intensity of subjective experience but not cardiac reactivity, and that intensity is associated with different regions of the anterior insula in the two groups - a finding with implications for the study of culture and somatic symptoms.

Some recent work is also beginning to show the relevance of such a multi-level approach to bipolar disorder. Hechtman and colleagues (2013) draw on the emerging field of cultural neuroscience to present a transdiagnostic approach to positive emotion regulation, with implications for the study of manic and depressive states. They outline a research agenda for this work that involves evidence from five sources: cultural context; behavior; neuroimaging; genetic polymorphisms; and psychophysiology. The result is both a framework through which one can understand the wide range of findings across levels of analysis pertaining to positive emotion regulation, and an agenda for future integrative research.

An important consequence of this kind of complex, multilevel perspective is that it forces researchers to consider the possibility that cultural-historical change might shape phenomena under study in important ways. We began this chapter with a brief historical overview of disordered mood, emphasizing Western and Chinese perspectives. One straightforward way of reading such histories would be as an ordered progression towards a better understanding of depression and bipolar disorder. There is some truth to this view: contemporary neuroscience surely has an advantage, both ontologically and pragmatically, over the classical Greek humoral theory. Yet shifts over time in the social meaning of these disorders, and the symptoms that are emphasized at particular historical moments, raise intriguing questions about whether 
mood disorders have changed over time (Shorter, 1992).

There is evidence to suggest that rates of depression have increased in the United States (Hidaka, 2012), although it is difficult to disentangle shifts in diagnostic practices, how depression is discussed, and actual rates of serious distress (Murphy, Laird, Monson, Sobol, \& Leighton, 2000). From 1991-2 to 2001-2, the prevalence of MDD among U.S. adults increased from $3.3 \%$ to $7.1 \%$ (Compton, Conway, Stinson, \& Grant, 2006). Similar shifts may be underway in China, affecting both depression rates and symptom presentation. In 1993, a mental health survey undertaken in seven regions of China reported surprising point-prevalence estimates of $0.05 \%$ for any affective disorder (Zhang, Shen, \& Li, 1998). A recent meta-analysis based on 17 studies from 2001 to 2010 estimated a point prevalence rate of $1.6 \%$ ( $\mathrm{Gu}$ et al., 2013). Similarly, the $0.1 \%$ rate of BD (Shen et al., 2006), discussed earlier, might be smaller than rates in several other countries, but is considerably higher than the rates of $0.003 \%$ to $0.009 \%$ reported between 1958 and 1979 (Lin, Kleinman, $\&$ Lin, 1981), and at least somewhat higher than the rates of $0.037 \%$ to $0.089 \%$ reported in the 1980 s (Cheung, 1991).

Given emerging evidence of increasing individualism and emotional expressivity in China as well (e.g., Cai, Kwan, \& Sedikides, 2011), we may be observing mental health consequences of striking - and rapid - changes in Chinese society. Another possibility is a shift in willingness to acknowledge serious distress coupled with a shift in presentation towards the psychological symptoms emphasized by Western research tools and training models. These speculations, however, must be tempered by acknowledgement that methodological issues plague comparison of studies conducted at different times (Guo, Tsang, Li, \& Lee, 2011). Future research in this area should move beyond observation of base rate differences to studies designed to evaluate temporal shifts and unpack underlying cultural-historical mechanisms.

\section{Concluding Remarks}

There is a growing research literature on the cultural shaping of depression, accompanied by an increasing number of studies that move beyond description to explanation. Among the latter, studies conducted in East Asian, and especially Chinese, contexts tend to predominate. The literature would be enhanced by increased attention to other cultural contexts. As for the cultural shaping of BD, considerably fewer studies have been conducted, but there has been an increasing attention in recent years to the potential role of the sociocultural environment. Unfortunately, much of this literature is limited by the uncritical adoption of Western-derived diagnostic criteria, and by the tendency to catalogue group differences rather than unpack the mechanisms and processes of cultural variation. We propose that the best way to advance this work in the future is for researchers interested in culturemind links to engage directly with researchers focused on mind-brain links, in order to develop a truly integrative science of mood disorders.

\section{References}

Abbey, S. E., \& Garfinkel, P. E. (1991). Neurasthenia and chronic fatigue syndrome. American Journal of Psychiatry, 148, 1638-1646.

Akiskal, H. S. (1996). The prevalent clinical spectrum of bipolar disorders: Beyond DSMIV. Journal of Clinical Psychopharmacology, 16, 4S-14S.

American Psychiatric Association. (2013). Diagnostic and statistical manual of mental disorders (5th Ed.). Washington, DC: Author.

Andrade, L., Caraveo-Anduaga, J. J., Berglund, P., Bijl, R. V., de Graaf, R., Vollebergh, W., .. \& \& Wittchen, H.-U. (2003). The epidemiology of major depressive episodes: Results from the International Consortium of Psychiatric Epidemiology (ICPE) surveys. International Journal of Methods in Psychiatric Research, 12, 3-21.

Auerbach, R. P., Abela, J. R. Z., Zhu, X., \& Yao, S. (2010). Understanding the role of coping in the development of depressive symptoms: Symptom specificity, gender differences, and cross-cultural applicability. British Journal of Clinical Psychology, 49, 547-561.

Auerbach, R. P., Eberhart, N. K., \& Abela, J. R. Z. (2010). Cognitive vulnerability to depression in Canadian and Chinese adolescents. Journal of Abnormal Child Psychology, 38, 57-68.

Ban, L. M., Kashima, Y., \& Haslam, N. (2010). Does understanding behavior make it seem normal? Perceptions of abnormality among Euro-Australians and ChineseSingaporeans. Journal of Cross-Cultural Psychology, 43, 286-298.

Beard, G. M. (1869). Neurasthenia, or nervous exhaustion. Boston Medical and Surgical Journal, 80, 217-221.

Bernstein, K. S., Lee, J. S., Park, S. Y., \& Jyoung, J. P. (2008). Symptom manifestations and expressions among Korean immigrant women suffering with depression. Journal of Advanced Nursing, 61, 393-402.

Berrios, G. E. (1996). The history of mental symptoms: Descriptive psychopathology since the nineteenth century Cambridge, U.K.: Cambridge University Press.

Cai, H., Kwan, V. S., \& Sedikides, C. (2012). A sociocultural approach to narcissism: The case of modern China. European Journal of Personality, 26, 529-535.

Carta, M. G., Moro, M. F., Kovess, V., Brasesco, M. V., Bhat, K. M., Angermeyer, M. C., \& Akiskal, H. S. (2012). Could hypomanic traits explain selective migration? Verifying the hypothesis by the surveys on Sardinian migrants. Clinical Practice and Epidemiology in Mental Health, 8, 175-179.

Caspi, A., Hariri, A. R., Holmes, A., Uher, R., \& Moffitt, T. E. (2010). Genetic sensitivity to the environment: The 
case of the serotonin transporter gene and its implications for studying complex diseases and traits. Focus, 8, 398-416.

Chatel, J. C., \& Peele, R. (1970). A centennial review of neurasthenia. American Journal of Psychiatry, 126, 1404-1413.

Chen, X., Rubin, K. H., \& Li, B. S. (1995). Depressed mood in Chinese children: Relations with school performance and family environment. Journal of Consulting and Clinical Psychology, 63, 938-947.

Chentsova-Dutton, Y. E., Chu, J. P., Tsai, J. L., Rottenberg, J., Gross, J. J., \& Gotlib, I. H. (2007). Depression and emotional reactivity: Variation among Asian Americans of East Asian descent and European Americans. Journal of Abnormal Psychology, 116, 776-785.

Cheung, F. (1991). Health psychology in Chinese societies in Asia. In: M. Jansen \& J. Wenman (Eds.). The international development of health psychology (pp. 63-74). Reading, U.K.: Harwood Academic Press.

Chiao, J. Y., \& Blizinsky, K. D. (2009). Culture-gene coevolution of individualism-collectivism and the serotonin transporter gene. Proceedings of the Royal Society B: Biological Sciences, 277, 529-537.

Compton, W. M., Conway, K. P., Stinson, F. S., \& Grant, B. F. (2006). Changes in the prevalence of major depression and comorbid substance use disorders in the United States between 1991-1992 and 2001-2002. American Journal of Psychiatry, 163, 2141-2147.

Demyttenaere, K., Bruffaerts, R., Lee, S., Posada-Villa, J., Kovess, V., Angermeyer, M. C., . . . \& Von Korff, M. (2007). Mental disorders among persons with chronic back or neck pain: Results from the World Mental Health Surveys. Pain, 129, 332-42.

Egeland, J. A., Hostetter, A. M., \& Eshleman, S. K. (1983) Amish Study, III: The impact of cultural factors on diagnosis of bipolar illness. American Journal of Psychiatry, 140, 67-71.

Ford, B. Q., Dmitrieva, J. O., Heller, D., ChentsovaDutton, Y., Grossmann, I., Tamir, M., .. \& \& Mauss, I. B. (2015). Culture shapes whether the pursuit of happiness predicts higher or lower well-being. Journal of Experimental Psychology, General. Published online before print. http://dx.doi.org/10/1037/xge0000108

Ford, B. Q., Mauss, I. B., \& Gruber, J. (2015). Valuing happiness is associated with bipolar disorder. Emotion, 15, 211-222.

Ford, B. Q., Shallcroft, A. J., Mauss, I. B., Floerke, V. A., \& Gruber, J. (2014). Desperately seeking happiness: Valuing happiness is associated with symptoms and diagnosis of depression. Journal of Social and Clinical Psychology, 33, 890-905.

Good, B. (1977). The heart of what's the matter: The semantics of illness in Iran. Culture, Medicine, and Psychiatry, $1,25-58$.
Grant, K. E., Compas, B. E., Thurm, A. E., McMahon, S. D., Gipson, P. Y., Campbell, A. J., . . . \& Westerholm, R. I. (2006). Stressors and child and adolescent psychopathology: Evidence of moderating and mediating effects. Clinical Psychology Review, 26, 257-283.

Gu, L., Xie, J., Long, J., Chen, Q., Chen, Q., Pan, R., ... $\& \mathrm{Su}$, L. (2013). Epidemiology of depression in mainland China: A systematic review. PLoS One, 8, e65356.

Guo, W.-J., Tsang, A., Li, T., \& Lee, S. (2011). Psychiatric epidemiological survey in China 1960-2010: How real is the increase of mental disorders? Current Opinion in Psychiatry, 24, 324-330.

Hechtman, L. A., Raila, H., Chiao, J. Y., \& Gruber, J. (2013). Positive emotion regulation and psychopathology: A transdiagnostic cultural neuroscience approach. Journal of Experimental Psychopathology, 4, 502-528.

Heine, S. J., Lehman, D. R., Markus, H. R., \& Kitayama, S. (1999). Is there a universal need for positive self-regard? Psychological Review, 106, 766-794.

Hesketh, T., Ding, Q. J., \& Jenkins, R. (2002). Suicide ideation in Chinese adolescents. Social Psychiatry and Psychiatric Epidemiology, 37, 230-235.

Hidaka, B. H. (2012). Depression as a disease of modernity: Explanations for increasing prevalence. Journal of Affective Disorders, 140, 205-214.

Hutchins, E. (2011). Enculturating the supersized mind. Philosophical Studies, 152, 437-446.

Immordino-Yang, M. H., Yang, X. F., \& Damasio, H. (2014). Correlations between socialemotional feelings and anterior insula activity are independent from visceral states but influenced by culture. Frontiers in Human Neuroscience, 8,728 .

Isaac, M., Janca, A., \& Orley, J. (1996). Somatization-a culture-bound or universal syndrome? Journal of Mental Health, 5, 219-222.

Jackson, S. W. (1985). Acedia the sin and its relationship to sorrow and melancholia. In A. Kleinman \& B. Good (Eds), Culture and depression (pp. 43-62). Berkeley \& Los Angeles, CA: University of California Press.

Jurcik, T., Chentsova-Dutton, Y. E., Solopieva-Jurcikova, L., \& Ryder, A. G. (2013). Russians in treatment: The evidence base supporting cultural adaptations. Journal of Clinical Psychology, 69, 774-791.

Kessler, R. C., Aguilar-Gaxiola, S., Alonso, J., Chatterji, S., Lee, S., Ormel, J., . . . \& Wang, P. S. (2009). The global burden of mental disorders: An update from the WHO World Mental Health (WMH) surveys. Epidemiologia E Psichiatria Sociale, 18, 23-33.

Kessler, R. C., McGonagle, K. A., Zhao, S., Nelson, C. B., Hughes, M., Eshleman, S., . . . \& Kendler, K. S. (1994). Lifetime and 12-month prevalence of DSM-III-R psychiatric disorders in the United States: Results from the National Comorbidity Survey. Archives of General Psychia- 
try, 51, 8-19.

Kim, H. S., Sherman, D. K., Sasaki, J. Y., Xu, J., Chu, T. Q., Ryu, C., . . . \& Taylor, S. E. (2010). Culture, distress, and oxytocin receptor polymorphism (OXTR) interact to influence emotional support seeking. Proceedings of the National Academy of Sciences, 107(36), 15717-15721.

Kirmayer, L. J. (2007). Psychotherapy and the cultural concept of the person. Transcultural Psychiatry, 44, 232-257.

Kirmayer, L. J., \& Sartorius, N. (2007). Cultural models and somatic syndromes. Psychosomatic Medicine, 69, 832-840.

Kirov, G., \& Murray, R. M. (1999). Ethnic differences in the presentation of bipolar affective disorder. European Psychiatry, 14, 199-204.

Kitayama, S., \& Uskul, A. K. (2011). Culture, mind, and the brain: Current evidence and future directions. Annual Review of Psychology, 62, 419-49.

Kleinman, A. (1982). Neurasthenia and depression: A study of somatization and culture in China. Culture, Medicine and Psychiatry, 6, 117-190.

Kleinman, A. (1986). Social origins of disease and distress: Depression, neurasthenia, and pain in modern China. New Haven, CT: Yale University Press.

Kohrt, B. A., Kunz, R. D., Baldwin, J. L., Koirala, N. R., Sharma, V. D., \& Nepal, M. K. (2005). "Somatization" and "comorbidity": A study of jhum-jhum and depression in rural Nepal. Ethos, 33, 125-147.

Koss-Chioino, J. D. (1999). Depression among Puerto Rican women: Culture, etiology and diagnosis. Hispanic Journal of Behavioral Sciences, 21, 330-350.

Kraepelin, E. (1921). Manic-depressive illness and paranoia. Edinburgh, U.K.: E. \& S. Livingstone.

Lee, S. (1999). Diagnosis postponed: Shenjing Shuairuo and the transformation of psychiatry in post-Mao China. Culture, Medicine, and Psychiatry, 23, 349-380.

Lee, S. (2001). From diversity to unity: The classification of mental disorders in 21st-century China. Psychiatric Clinics of North America, 24, 421-431.

Lee, H. Y., Lytle, K., Yang, P. N., \& Lum, T. (2010). Mental health literacy in Hmong and Cambodian elderly refugees: A barrier to understanding, recognizing, and responding to depression. International Journal of Aging $\mathcal{E}$ Human Development, 71, 323-344.

Li, H., \& Zhang, Y. (2008). Factors predicting rural Chinese adolescents' anxieties, fears and depression. School Psychology International, 29, 376-384.

Lin, E. H., \& Von Korff, M. (2008). Mental disorders among persons with diabetes - results from the world mental health surveys. Journal of Psychosomatic Research, 65, 571-580.

Lin, K., Kleinman, A., \& Lin, T. (1981). Overview of mental disorders in Chinese cultures: Review of epidemi- ological and clinical studies. In T. Lin \& A. Kleinman (Eds.), Normal and abnormal behavior in Chinese culture (pp. 237-272). Dordrecht, The Netherlands: Springer.

Liu, S. (1989). Neurasthenia in China: Modern and traditional criteria for its diagnosis. Culture, Medicine and Psychiatry, 13, 163-186.

Lopez, A. D., \& Mathers, C. D. (2006). Measuring the global burden of disease and epidemiological transitions: 2002-2030. Annals of Tropical Medicine and Parasitology, 100, 481-499.

Makanjuola, R. O. (1985). Recurrent unipolar manic disorder in the Yoruba Nigerian: Further evidence. The British Journal of Psychiatry, 147, 434-437.

Markus, H. R., \& Kitayama, S. (1991). Culture and the self: Implications for cognition, emotion, and motivation. Psychological Review, 98, 224-253.

Markus, H. R., \& Kitayama, S. (2010). Cultures and selves: A cycle of mutual constitution. Perspectives on Psychological Science, 5, 420-430.

Mauss, I. B., Savino, N. S., Anderson, C. L., Weisbuch, M., Tamir, M., \& Laudenslager, M. L. (2012). The pursuit of happiness can be lonely. Emotion, 12, 908-912.

Mauss, I. B., Tamir, M., Anderson, C. L., \& Savino, N. S. (2011). Can seeking happiness make people unhappy? Paradoxical effects of valuing happiness. Emotion, 11, 807-815.

Murphy, J. M., Laird, N. M., Monson, R. R., Sobol, A. M., \& Leighton, A. H. (2000). A 40-year perspective on the prevalence of depression: The Stirling County Study. Archives of General Psychiatry, 57, 209-215.

Murray, C. J., \& Lopez, A. D. (1996). The global burden of disease, vol. 1. Cambridge, MA: Harvard University Press.

Noaghiul, S., \& Hibbeln, J. R. (2003). Cross-national comparisons of seafood consumption and rates of bipolar disorders. American Journal of Psychiatry, 160, 2222-2227.

O'Connor, L. E., Berry, J. W., Weiss, J., \& Gilbert, P. (2002). Guilt, fear, submission, and empathy in depression. Journal of Affective Disorders, 71, 19-27.

Ohayon, M. M., \& Hong, S. (2006). Prevalence of major depressive disorder in the general population of South Korea. Journal of Psychiatric Research, 40, 30-36.

Osher, Y., Yaroslavsky, Y., El-Rom, R., \& Belmaker, R. H. (2000). Predominant polarity of bipolar patients in Israel. World Journal of Biological Psychiatry, 1, 187-189.

Parker, G., Chan, B., Tully, L., \& Eisenbruch, M. (2005). Depression in the Chinese: The impact of acculturation. Psychological Medicine, 35, 1475-1483.

Parker, G., Cheah, Y., \& Roy, K. (2001). Do the Chinese somatize depression? A cross-cultural study. Social Psychiatry and Psychiatric Epidemiology, 36, 287-293.

Pritzker, S. (2007). Thinking hearts, feeling brains: Metaphor, culture, and the self in Chinese narratives of depression. Metaphor and Symbol, 22, 251-274. 
Reynolds, J., Stewart, M., MacDonald, R., \& Sischo, L. (2006). Have adolescents become too ambitious? High school seniors' educational and occupational plans, 1976 to 2000. Social Problems, 53, 186-206.

Ryder, A. G., \& Chentsova-Dutton, Y. E. (2015). Cultural-clinical psychology: From cultural scripts to contextualized treatments. In L. J. Kirmayer, R. Lemelson, \& C. A. Cummings (Eds.), Re-visioning psychiatry: Cultural phenomenology, critical neuroscience, and global mental health (pp. 400-433). Cambridge, U.K.: Cambridge University Press.

Ryder, A., G. \& Chentsova-Dutton, Y. E. (2012). Depression in Cultural Context: "Chinese Somatization," Revisited. Psychiatric Clinics of North America, 35, 15-36.

Ryder, A. G., Yang, J., Zhu, X., Yao, S., Yi, J., Heine, S. J., \& Bagby, R. M. (2008). The cultural shaping of depression: Somatic symptoms in China, psychological symptoms in North America? Journal of Abnormal Psychology, 117, 300-313.

Sastry, J., \& Ross, C. E. (1998). Asian ethnicity and the sense of personal control. Social Psychology Quarterly, 61, 101-120.

Sethi, S., \& Khanna, R. (1993). Phenomenology of mania in eastern India. Psychopathology, 26, 274-278.

Shen, Y., Zhang, M., Huang, Y., He, Y., Liu, Z., Cheng, H., . . . \& Kessler, R. C. (2006). Twelvemonth prevalence, severity, and unmet need for treatment of mental disorders in metropolitan China. Psychological Medicine, 36, 257-267.

Shorter, E. (1992). From paralysis to fatigue: A history of psychosomatic medicine in the modern era. New York, NY: Free Press.

Shweder, R. A. (1990). Cultural psychology: What is it? In J. Stigler, R. Shweder, \& G. Herdt (Eds.), Cultural Psychology: Essays on Comparative Human Development (1-43). Cambridge, U.K.: Cambridge University Press.

Simon, G. E., VonKorff, M., Piccinelli, M., Fullerton, C., \& Ormel, J. (1999). An international study of the relation between somatic symptoms and depression. New England Journal of Medicine, 341, 1329-1335.

Sperber, D., \& Hirschfeld, L. A. (2004). The cognitive foundations of cultural stability and diversity. Trends in Cognitive Sciences, 8, 40-46.

Swinnen, S. G., \& Selten, J. P. (2007). Mood disorders and migration meta-analysis. British Journal of Psychiatry, 190, 6-10.

Stewart, S. M., Kennard, B. D., Lee, P. W., Hughes, C. W., Mayes, T. L., Emslie, G. J., \& Lewinsohn, P. M. (2004). A cross-cultural investigation of cognitions and depressive symptoms in adolescents. Journal of Abnormal Psychology, $113,248-257$.

Tseng, W.-S. (1974). The development of psychiatric concepts in Chinese medicine. Archives of General Psychiatry, 29, 569-575.

Turvey, C. L., Jogerst, G., Kim, M. Y., \& Frolova, E. (2012). Cultural differences in depressionrelated stigma in late-life: A comparison between the USA, Russia, and South Korea. International Psychogeriatrics, 24, 1642-1647.

Vasiliadis, H., Lesage, A., Adair, C., Wang, P. S., \& Kessler, R. C. (2007). Do Canada and the United States differ in prevalence of depression and utilization of services? Psychiatric Services, 58, 63-71.

Wenzel, S. (1960). The sin of sloth: Acedia in medieval thought and literature. Chapel Hill, NC: University of North Carolina Press.

World Health Organization. (2008). ICD-10: International statistical classification of diseases and related health problems (10th Rev. ed.). New York, NY: Author.

Yen, S., Robins, C. J., \& Lin, N. (2000). A cross-cultural comparison of depressive symptom manifestation: China and the United States. Journal of Consulting and Clinical Psychology, 68, 993-999.

Zhang, W. X., Shen, Y. C., \& Li, S. R. Epidemiological investigation on mental disorders in 7 areas of China. Chinese Journal of Psychiatry, 31, 69-71. 